\title{
Predoctoral Dental Student Evaluation of American Academy of Pediatric Dentistry's Caries-Risk Assessment Tool
}

\author{
S.M. Hashim Nainar, B.D.S., M.D.Sc.; Lloyd H. Straffon, D.D.S., M.S. \\ Abstract: The purpose of this study was to determine predoctoral dental student evaluation of the American Academy of Pediatric \\ Dentistry's Caries-Risk Assessment Tool (CAT) for children. Dental students were introduced to the CAT instrument as part of \\ their didactic pediatric dentistry curriculum. These students were later encouraged to use the CAT instrument for determining \\ caries risk in their pediatric patients. Following a two-year exposure to the CAT instrument, dental students completed an \\ anonymous seven-item evaluation of the instrument at the end of their primary clinical experience in pediatric dentistry. The \\ students were asked to score each item on a five-point scale ranging from strongly agree (\#5) to neutral (\#3) to strongly disagree \\ (\#1). Ninety-seven percent of eligible students completed the CAT evaluation questionnaire. Most students agreed that the CAT \\ instrument was easy to understand (86 percent), simple to apply (76 percent), useful for prescribing radiographs (76 percent), and \\ useful for determining preventive procedures ( 84 percent). Eighty percent of them indicated that they were likely to use the CAT \\ instrument in their clinical practice. In conclusion, student acceptance of the CAT instrument indicates that it may educate \\ predoctoral dental students regarding caries risk assessment in children.
}

Dr. Nainar is Clinical Associate Professor and Dr. Straffon is Professor-both in the Department of Orthodontics and Pediatric Dentistry, School of Dentistry, University of Michigan. Direct correspondence and requests for reprints to Dr. S.M. Hashim Nainar, Department of Orthodontics and Pediatric Dentistry, School of Dentistry, University of Michigan, Ann Arbor, MI 481091078; 734-763-2331 phone; 734-615-7294 fax; nainar@umich.edu.

Key words: dental education, pediatric dentistry, dental caries susceptibility

Submitted for publication 8/8/05; accepted 10/21/05

$\mathrm{S}$ ince the 1970s, there has been a decline in dental caries levels in U.S. children. ${ }^{1,2}$ Despite the general decline in pediatric caries experience, it has been observed that "dental caries remains the single most common disease of childhood that is neither self-limiting nor amenable to short-term pharmacological management." ${ }^{\prime 3}$ In recent times, dental caries experience has become concentrated in a segment of the pediatric population, with 80 percent of the caries experienced in permanent teeth occurring in one-quarter of five-to-seventeen-year-old children. ${ }^{4}$ This concentration of dental caries experience in a minority of the children provides one basis for the use of caries risk assessment in clinical practice.

Another reason for caries risk assessment is provided by the caries balance concept of caries management. ${ }^{5}$ According to the caries balance concept, dental caries is a dynamic disease process involving pathological factors causing demineralization, which are counterbalanced by protective factors promoting remineralization. ${ }^{5}$ The theory that dental caries disease process occurs on a reversible continuum of demineralization until eventual irreversible cavitation emphasizes the importance of early detection and consequent intervention to potentially reverse the disease process. ${ }^{6}$ This in turn reinforces the need for caries risk assessment to stratify and determine clinical management of individuals based on their risk determination. ${ }^{5}$ It has been observed that, in the future, "tooth restorations will become less and less desirable as a treatment and will be used only as a final resort" when intervention measures have not been successful in managing dental caries disease activity. ${ }^{5}$ Risk-based management of dental caries has been shown to be a viable and practical approach. ${ }^{7,8}$

The two reasons (dental caries concentrated in 25 percent of children and caries balance concept) described above underscore the importance of having predoctoral dental students obtain an understanding of the concept of caries risk assessment in children. Imparting knowledge of pediatric caries risk assessment to future dental practitioners has two goals:

1. provide efficient and targeted delivery of preventive services to children who will benefit the most from caries prevention; and

2. promote the concept of minimally invasive dentistry in clinical practice as a new generation of dental practitioners assumes the mantle of clinical practice.

Accomplishment of these goals provides justification for educating predoctoral dental students in 
caries risk assessment. Review of the literature, however, indicates that there is no definitive formula available for caries risk assessment in individual patients. ${ }^{5}$ Instead contemporary caries risk assessment is based upon clinical judgment, i.e., the application of inductive and deductive reasoning to assess multiple caries risk factors rather than upon a precise formula-driven science. ${ }^{9}$

In April 2002, the American Academy of Pediatric Dentistry (AAPD) sponsored the Pediatric Restorative Dentistry Consensus Conference wherein caries risk indicators in children were enumerated and classified by risk stratification. ${ }^{10}$ Later that year, AAPD adopted the Caries-Risk Assessment Tool (CAT) for determining caries risk in children. ${ }^{11}$ This new instrument has the potential to further promote understanding and application of pediatric caries risk assessment in clinical practice.

The objective of our study was to determine predoctoral dental student evaluation of the American Academy of Pediatric Dentistry's Caries-Risk Assessment Tool (CAT) for children.

\section{Methods}

The Caries-Risk Assessment Tool (CAT) was introduced during the 2002 fall term to predoctoral dental students (Class of 2005) in their sophomore year at the University of Michigan School of Dentistry as they began their formal introduction to pediatric dentistry. This class received one formal fiftyminute lecture on caries risk assessment in children. As part of their didactic curriculum, these sophomore dental students completed case-based written examinations regarding their knowledge and application of the CAT instrument.

The predoctoral dental curriculum at the University of Michigan introduced direct patient care in pediatric dentistry during the sophomore year. However, the primary clinical experience in pediatric dentistry occurred during two block rotations in the junior year. During their pediatric block rotations, those students in the Class of 2005 reviewed the cariesrisk status of their patients with their assigned clinical faculty. This allowed the supervising clinical faculty to make changes if necessary and provide an explanation for modifying the student's caries risk assessment.

Upon completion of their final block rotation in pediatric dentistry, junior dental students attended an exit seminar wherein their overall pediatric den- tistry clinical rotation experience was discussed. During this exit seminar, the students completed an anonymous evaluation of their pediatric clinical rotation. This one-page pediatric clinical rotation evaluation questionnaire had eight questions; five of them were measured on a five-point scale, while the remaining three were short answer questions.

The Class of 2005 completed their second pediatric dentistry block rotation in groups over the time period October 2003 to April 2004. In addition to the usual evaluation of the pediatric clinical rotation, these junior dental students were also asked to complete an anonymous evaluation of the CAT instrument. The format of the CAT evaluation questionnaire was structured similar to that of the rotation evaluation questionnaire. The one-page CAT evaluation questionnaire had seven questions measured on a five-point scale as follows: strongly agree $=5$; agree $=4$; neutral $=3$; disagree $=2$; strongly disagree $=1$.

The seven questions evaluating the Caries-Risk Assessment Tool were:

Q1. The Caries-Risk Assessment Tool was easy to understand.

Q2. The Caries-Risk Assessment Tool was simple to apply in the pediatric dentistry clinic.

Q3. The Caries-Risk Assessment Tool was useful for prescribing radiographs in children.

Q4. The Caries-Risk Assessment Tool was useful for determining preventive procedures in children.

Q5. The faculty was helpful in using the CariesRisk Assessment Tool with my patients in the pediatric dentistry clinic.

Q6. The faculty and I agreed on my rating of caries risk using the Caries-Risk Assessment Tool in the pediatric dentistry clinic.

Q7. I will likely use the Caries-Risk Assessment Tool in my clinical practice.

For purposes of analysis, students' ratings \#5 (strongly agree) and \#4 (agree) were combined into a single "agree" rating. Frequency distribution analysis was performed for the data set.

This study was reviewed and considered exempt by the Health Sciences Institutional Review Board at the University of Michigan.

\section{Results}

Data collection for the survey was conducted from October 2003 through April 2004 and yielded one hundred and four responses ( 97 percent) from a 
junior class of 107 students. All respondents completed all seven items of the study questionnaire, and there were no missed responses.

Most of the students agreed (ratings \#4 and \#5) with all seven items of the study questionnaire. The students' agreement ratings ranged from a maximum of 86 percent for ease in understanding the CAT instrument to a minimum of 74 percent for the faculty's helpfulness in using the CAT instrument for their patients in the pediatric dentistry clinic (Table 1).

None of the students selected strong disagreement (rating \#1) as a response on the five-point scale with any of the seven items in the study questionnaire. Less than 6 percent of students selected disagreement (rating \#2) for any of the seven items on the questionnaire.

\section{Discussion}

This study measured predoctoral dental students' evaluation of the American Academy of Pediatric Dentistry's Caries-Risk Assessment Tool. The sample of dental students included in this study received one formal fifty-minute didactic lecture in caries risk assessment in children as part of their regular pediatric dental curriculum followed by completion of their primary clinical experiences in pediatric dentistry during the sophomore and junior years.

It has been observed that "caries risk indicators may be useful in the clinical management of dental caries by helping dental professionals to determine if additional diagnostic procedures are required; identify patients who require caries control measures; assess the impact of these measures; guide in treatment planning decisions; and determine the timing of re- call appointments." ${ }^{\prime 2}$ The results of the survey completed by predoctoral dental students indicate that the AAPD's CAT instrument may be a useful pedagogical tool for teaching pediatric caries risk assessment. Junior dental students' evaluation of the CAT instrument was overwhelmingly positive with regard to its ease of learning and application in children.

The CAT instrument was well received by the dental student cohort in our study, but it does have its limitations. The instrument was meant to be used both by dental and nondental personnel and therefore has been somewhat oversimplified. This in particular impacts upon the classification of risk in a child following evaluation of the various caries risk indicators. The CAT instrument notes that "each child's ultimate risk classification is determined by the highest risk category where a risk indicator exists" (i.e., the presence of a single risk indicator in any area of the "high-risk" category is sufficient to classify a child as being at "high risk"). ${ }^{11}$ The CAT instrument, therefore, is extremely sensitive with a greater likelihood to incorrectly increase caries risk stratification of individual children. For instance, children from low-income families who are covered by the Medicaid program would be considered as being at high caries-risk according to the CAT instrument even though individual children might be caries-free without increased risk for dental caries.

Despite the abovementioned classification flaw of the CAT instrument, it appears to be a viable tool for clinical practitioners. Our study has demonstrated that the CAT instrument is likely to be well received by clinical practitioners. It has been designed to be sensitive to various aspects of caries risk assessment in children even as its format remains simple and easy to understand. The University of North Caro-

Table 1. Dental students' evaluation of Caries-Risk Assessment Tool (CAT)*

Caries-Risk Assessment Tool evaluation

Students' rating $(n=104)$

\begin{tabular}{|c|c|c|c|}
\hline & Agree & Neutral & Disagree \\
\hline Easy to understand & $86 \%$ & $14 \%$ & 0 \\
\hline Simple to apply & $76 \%$ & $22 \%$ & $2 \%$ \\
\hline Useful for prescribing radiographs & $76 \%$ & $20 \%$ & $4 \%$ \\
\hline Useful for determining preventive procedures & $84 \%$ & $12 \%$ & $4 \%$ \\
\hline Faculty were helpful with using CAT & $74 \%$ & $20 \%$ & $6 \%$ \\
\hline Faculty agreed with my rating of risk determined using CAT & $79 \%$ & $19 \%$ & $2 \%$ \\
\hline Likely to use CAT in my clinical practice & $80 \%$ & $16 \%$ & $4 \%$ \\
\hline
\end{tabular}

*American Academy of Pediatric Dentistry. Policy on the use of a caries-risk assessment tool (CAT) for infants, children, and adolescents (Reference Manual 2002-03). Pediatr Dent 2002;24(7):15-17. 
lina Caries-Risk Assessment Study has shown that dentist prediction, i.e., "personal clinical judgment," was a strong predictor of future caries increment in children. ${ }^{13}$ Any pedagogical instrument, therefore, that provides an alternate for experiential learning in caries risk assessment would be of value to novice dental practitioners. Use of the CAT instrument may help to familiarize novice dental practitioners with the concept of pediatric caries risk assessment and thereby improve their clinical judgment in determining a child's caries-risk status.

\section{Conclusion}

Student acceptance of the American Academy of Pediatric Dentistry's Caries-Risk Assessment Tool indicates that it may educate predoctoral dental students regarding caries risk assessment in children.

\section{REFERENCES}

1. Oral health of United States children: the national survey of dental caries in U.S. school children, 1986-87. National Institute of Dental Research. NIH Publication No. 892247, September 1989.

2. Brown LJ, Wall TP, Lazar V. Trends in total caries experience: permanent and primary teeth. J Am Dent Assoc 2000;131:223-31.

3. Vargas CM, Crall JJ, Schneider DA. Sociodemographic distribution of pediatric dental caries: NHANES III, 19881994. J Am Dent Assoc 1998;129:1229-38.
4. Kaste LM, Selwitz RH, Oldakowski RJ, Brunelle JA, Winn DM, Brown LJ. Coronal caries in the primary and permanent dentition of children and adolescents 1-17 years of age: United States, 1988-1991. J Dent Res 1996; 75(Spec Iss):631-41.

5. Featherstone JDB. The science and practice of caries prevention. J Am Dent Assoc 2000;131:887-99.

6. Featherstone JDB. The continuum of dental caries: evidence for a dynamic disease process. J Dent Res 2004;83(Spec Iss C):C39-42.

7. Pienihakkinen K, Jokela J. Clinical outcomes of risk-based caries prevention in preschool-aged children. Community Dent Oral Epidemiol 2002;30:143-50.

8. Bader JD, Shugars DA, Kennedy JE, Hayden WJ Jr, Baker S. A pilot study of risk-based prevention in private practice. J Am Dent Assoc 2003;134:1195-202.

9. Anderson M. Risk assessment and epidemiology of dental caries: review of the literature. Pediatr Dent 2002; 24:377-85.

10. Tinanoff N, Douglass JM. Clinical decision making for caries management in children. Pediatr Dent 2002;24: 386-92.

11. American Academy of Pediatric Dentistry. Policy on the use of a Caries-Risk Assessment Tool (CAT) for infants, children, and adolescents (Reference Manual 2002-03). Pediatr Dent 2002;24(7):15-7.

12. Zero D, Fontana M, Lennon AM. Clinical applications and outcomes of using indicators of risk in caries management. J Dent Educ 2001;65:1126-32.

13. Disney JA, Graves RC, Stamm JW, Bohannan HM, Abernathy JR, Zack DD. The University of North Carolina caries-risk assessment study: further developments in caries risk prediction. Community Dent Oral Epidemiol 1992;20:64-75. 